\title{
Plataforma robótica teleoperada para el Mercury Remote Robot Challenge
}

\section{Resumen}

El presente artículo expone el proceso que el semillero de investigación electrocun, de la Corporación Unificada Nacional de Educación Superior (CUN), llevó a cabo para diseñar y construir los robots teleoperados "Socco" y "Yipao" que participaron en el Mercury Remote Robot Challenge, en la Universidad de Oklahoma, Estados Unidos. En este sentido, en este documento se describe, de un lado, el proceso de diseño de las plataformas mecánicas y sistema electrónico, las cuales debían ser capaz de superar los retos propuestos por el concurso, y, del otro, la manera como se construyeron y ensamblaron para cumplir con las condiciones físicas de la pista de competencia.[

\section{Abstract}

This paper exposes the process that the ELECTROCUN research hotbed, of the Corporación Nacional Unificada de Educación Superior (CUN), carried out to design and build the "Socco" and "Yipao" teleoperated robots that participated in the Mercury Remote Robot Challenge, at the University of Oklahoma, United States. In this sense, this document describes, on the one hand, the design process of the mechanical platforms and electronic system, which should be able to overcome the challenges proposed by the tender, and, on the other, the way they were built and assembled to meet the physical conditions of the competition track.
Cómo citar este artículo (APA): Pinzón, S., Medina, A., Díaz, S. (2019). Plataforma robótica teleoperada para el Mercury Remote Robot Challenge. Hashtag, 14, 51-66.

> Palabras clave: robot, robot autónomo, robot teleoperado, sistema electrónico

> Keywords: autonomous robot, electronic system, robot, teleoperated robot

* Docente de Ingeniería Electrónica en la Corporación Unificada Nacional de Educación Superior. Contacto: said_pinzon@cun.edu.co

** Docente de Ingeniería Electrónica en la Corporación Unificada Nacional de Educación Superior. Contacto: alex_medinacel@cun.edu.co *** Docente de Ingeniería Electrónica en la Corporación Unificada Nacional de Educación Superior. Contacto: sergio_diaz@cun.edu.co 


\section{Introducción}

ctualmente, escuchar el término robot es
algo cotidiano; sin embargo, muchas veces las personas no saben que los robots son el resultado de la combinación de sistemas mecánicos con sistemas electrónicos que interactúan entre sí para cumplir con funciones específicas. El desarrollo de los robots ha aumentado como consecuencia del gran avance tecnológico que se ha venido manifestando a lo largo de los años $\mathrm{y}$, hoy en día, es posible observar en detalle los avances investigativos en el campo de la robótica. Sin duda, el software especializado ha logrado ayudar bastante a los avances presentes: este se implementa en los robots, debido a que estas son instrucciones que sigue cada robot para realizar una tarea específica o un listado de tareas programadas (Puentes, Ríos, y Salvador, 2010).

Toda vez que los humanos siempre han buscado simplificar sus tareas cotidianas (para ahorrar tiempo y esfuerzo), la robótica ha sido una justificación principal para el desarrollo de actividades donde un robot puede desempeñar un mejor papel que la solución hasta ese momento existente. Un ejemplo claro de esto es el automóvil: en un comienzo, para desplazarse a través de grandes distancias, el humano debía caminar durante mucho tiempo para llegar a su destino; después, empezó a utilizar tracción animal y era transportado en conjunto con algunos objetos más; luego, siguió innovando hasta ser capaz de crear vehículos que le permitían desplazarse más rápido y con un esfuerzo mucho menor, y, finalmente, en el siglo XXI, ya existen sistemas de desplazamiento autónomos $\mathrm{u}$ operados a distancia.
Estos últimos, los vehículos operados a distancia, son controlados desde una estación remota por un usuario. En principio, estos vehículos fueron creados con fines recreativos (carros a radiocontrol) y de entretenimiento, pero, debido a su gran potencial, desde hace varios años se han empezado a desarrollar sistemas teleoperados más robustos que han tenido implicaciones militares, civiles, gubernamentales e, incluso, sociales. Estos robots teledirigidos cuentan con equipos con cámaras, sensores, actuadores y sistemas de comunicación.

Ahora mismo, se pueden encontrar muchos vehículos teleoperados que, según su categorización, han sido diseñados para cumplir con diferentes tareas específicas. Por ejemplo, los vehículos militares no tripulados cuentan, de un lado, con funciones que les permiten explorar campos minados y lugares peligrosos (donde el ser humano no ha podido entrar o resulta peligrosa su incursión) y, del otro, con características físicas adaptadas especiales para el campo en el que se desarrollan (blindaje especial que lo protege de explosiones o posibles ataques). A continuación, se evidencian algunos de los vehículos teleoperados más relevantes de la actualidad:

- El Gladiator Tactital Unmanned Ground Vehicle (figura 1), que puede ser operado a una distancia de 80 kilómetros, apoya al cuerpo de marina de los Estados Unidos en misiones terrestres de alto riesgo. Sus funciones se basan en el uso bélico de su artillería, ya que puede realizar tareas de exploración, vigilancia y apertura de rutas obstaculizadas. También cuenta con cámaras de capacidad visual día - noche y térmica. 
Figura 1. Gladiator Tactical Unmanned Ground Vehicle - vehículo teleoperado del cuerpo de marina de Estados Unidos.

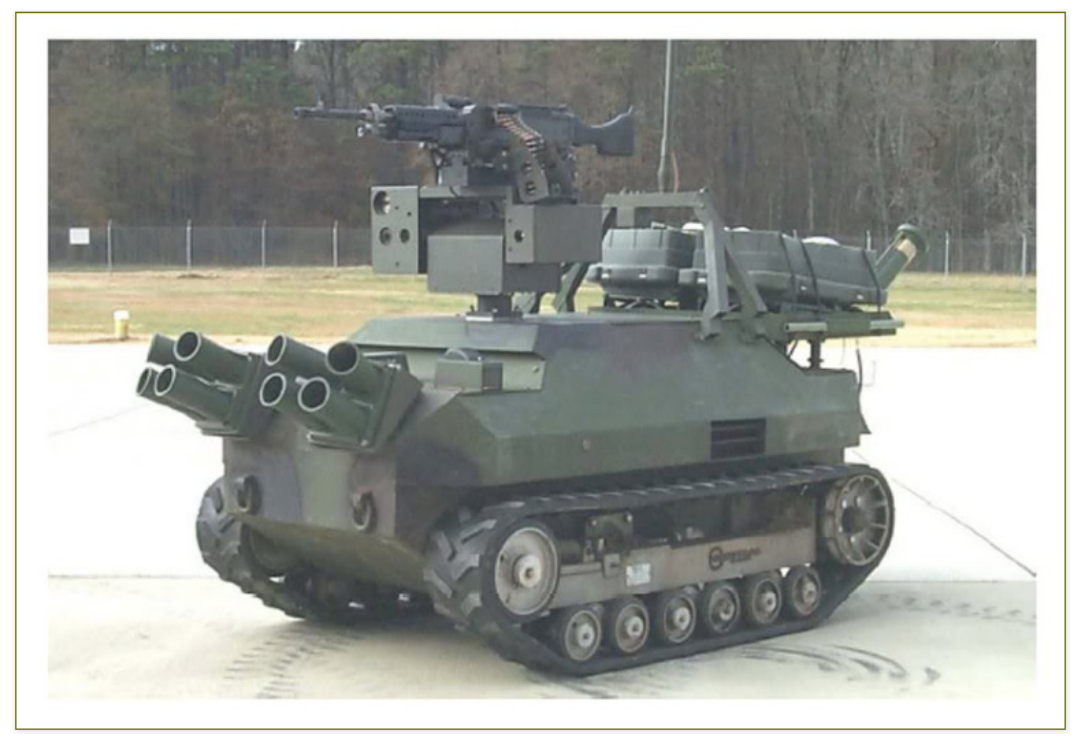

Fuente: Global Security (s. f.).

- El robot Multiscope 3D (figura 2) se utiliza para el rescate de personas en situaciones de catástrofe: según el tipo de situación que se esté presentando, el vehículo puede ser equipado con diferentes sistemas de ayuda, como lo son una manguera de agua, discos de corte, brazos robóticos, pala excavadora, etc. Este robot puede ser utilizado a una distancia de 90 kilómetros (Milirobotics, s. f.).

Figura 2. Multiscope 3D - vehículo de rescate teleoperado.

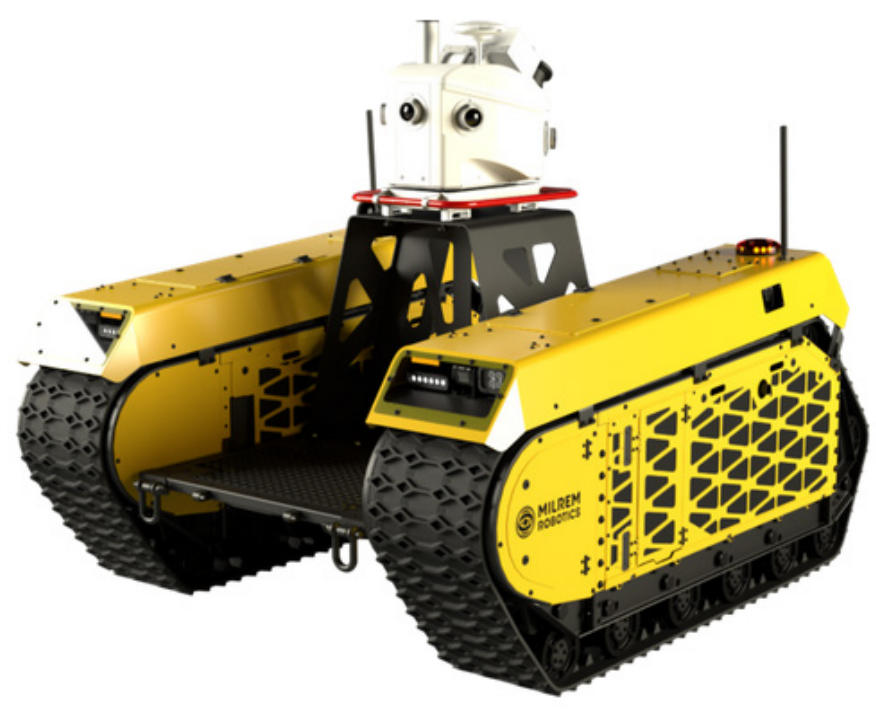

Fuente: Milrem Robotics (s. f.). 
- Otro tipo de vehículo teleoperado que actualmente tiene una gran acogida es el dron o Unmanned Aerial Vehicule (UAv). Este sistema robótico aéreo posee numerosas ventajas en el desplazamiento al compararlo con los vehículos no tripulados terrestres, lo que ha hecho que sea ampliamente utilizado a nivel comercial, civil y militar. Gracias a sus características, es altamente adaptable: puede llevar cámaras (vigilancia), brazos robóticos para el muestreo de objetos, compartimientos para el transporte de cargas, etc.
Por ejemplo, en el campo militar, los ejércitos utilizan drones para realizar vuelos periódicos sobre zonas de conflicto; en la agricultura, se equipan con equipos especiales y ayudan en la detección del estado del suelo y los cultivos; en aplicaciones comerciales, su uso permite que las entregas de productos sean mucho más rápidas, $\mathrm{y}$, en estudios fotométricos, se han integrado para determinar el uso de espacio de una edificación que se construirá.

Figura 3. Distintos tipos de drones.

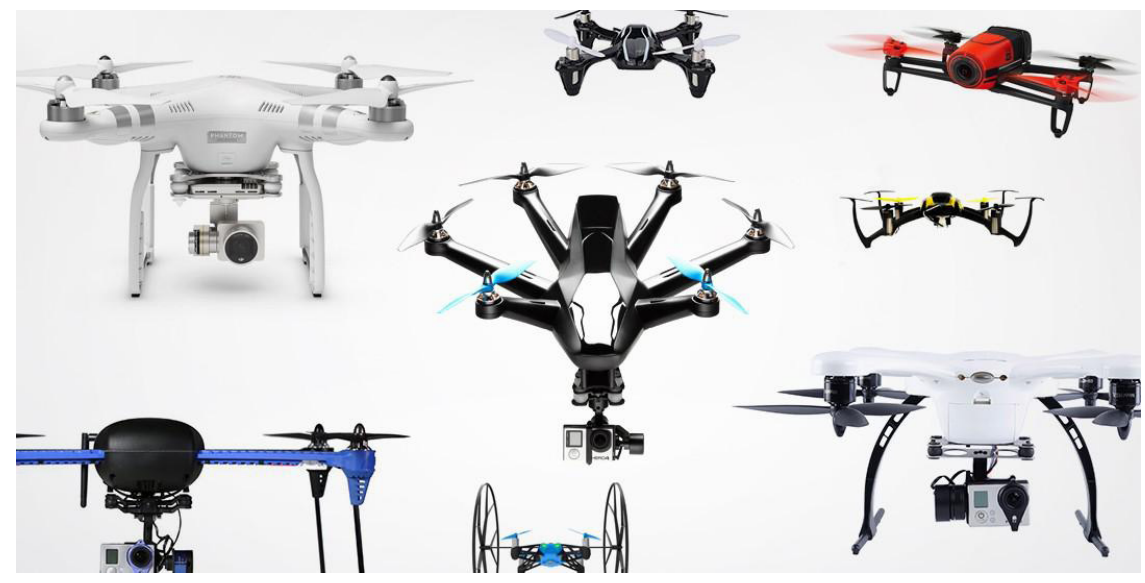

Fuente: Prometec (s. f.).

\section{Mercury Remote Robot Challenge}

La meta principal del Mercury Remote Robot Challenge es conducir un robot teleoperado (a una distancia de 80 kilómetros)m a través de un circuito con diferentes retos que exigen un alto nivel de calidad académica y profesional de los participantes en el desarrollo de plataformas robóticas que solucionen tareas específicas. Los equipos son conformados por tres personas con los siguientes roles:

- Piloto: es quien manipula al robot; se debe encontrar en un lugar a 80 kilómetros del robot.
- Mecánico: es quien manipula el robot físicamente; se encuentra en el lugar de la competencia y es la única persona del equipo autorizada para hacer ajustes al robot (si este lo requiere).

- Auxiliar mecánico: se encuentra al lado del mecánico, pero no puede manipular el robot. Únicamente puede asesorar al mecánico.

Los equipos que compiten tienen la presión de llevar a cabo el recorrido de la pista en el menor 
tiempo posible, superando los retos y acumulando puntos por cada obstáculo superado, y evitando cometer infracciones. Para teleoperar el vehículo, el equipo es libre de elegir, de un lado, el sistema de comunicación por utilizar, ya sea con módulos wifi, bluetooth, Zigbee, RF, etc., $\mathrm{y}$, del otro, la electrónica aplicada al robot para generar el desplazamiento.

Un detalle importante del concurso es que se entrega puntuación adicional al robot que cumpla con algunos retos de forma autónoma, para lo cual es necesario desarrollar un sistema electrónico adicional que lo permita.

\section{Pista}

La pista del concurso es un camino con un ancho de 60 centímetros que contiene múltiples obstáculos que los robots deben superar para continuar (ver figura 4):

Figura 4. Pista del Mercury Remote Robot Challenge.

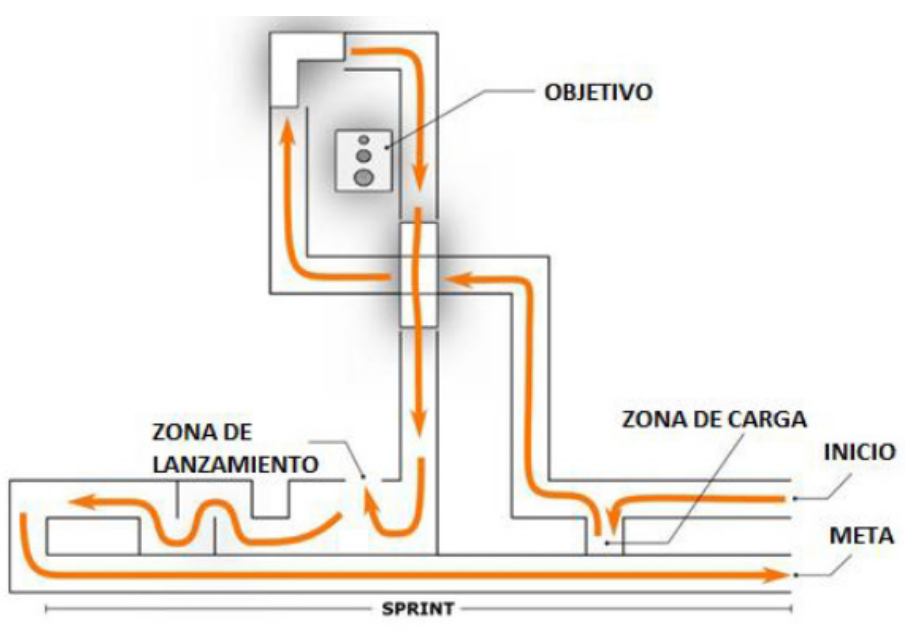

Fuente: elaboración propia.

\section{Prototipo}

De acuerdo con las especificaciones del concurso y con los requerimientos para el desarrollo de la plataforma robótica, se realiza un plan metodológico de tres fases en el que se evidencia el desarrollo del robot teleoperado. La fase 1 está compuesta por el diseño que se llevó a cabo para elaborar el robot de competencia y se divide en dos, sistema mecánico y sistema electrónico. En la primera parte, de acuerdo con las especificaciones de la pista, es importante aclarar que se presenta una tabla de ponderación entre diferentes diseños mecánicos que se ajustan a los desafíos propuestos en el concurso. Por su parte, en la segunda parte de la fase 1 , se expone el sistema electrónico propuesto, el cual debe ser capaz de cumplir con las demandas necesitadas para los obstáculos de pista; además, en una tabla de ponderaciones se evalúan los sensores necesarios para el desarrollo del hardware electrónico.

La fase 2 del proyecto se concentra en la construcción del prototipo. En este momento se construyen el sistema mecánico modelado en la fase 
1 y el sistema electrónico ajustado en la fase de diseño a las demandas de los sensores y actuadores presentes en el robot.

La fase 3 consiste en la evaluación de la plataforma robótica realizada; en este punto, se

\section{Desarrollo del proyecto}

\section{Fase 1. Diseño de la plataforma robótica}

\section{Primera parte (diseño mecánico)}

De acuerdo con las especificaciones de la pista y las reglas en general del concurso, se empieza a realizar un modelamiento de la estructura mecánica que debe de llevar el robot. De este modo, el grupo de trabajo del semillero ELECTROCUN diseña una tabla de prioridad donde se evalúan los aspectos más importantes con los que debía contar en el robot teleoperado. Es importante destacar que, para el Mercury Remote Robot Challenge, era esencial el tiempo en el que el robot recorre toda la pista y supera los obstáculos; por lo tanto, se define el tiempo como primera variable esencial que el robot debía cumplir. realiza un análisis de los resultados observados del robot en pista, su comportamiento en el enfrentamiento de los obstáculos y, por último, las conclusiones del proyecto realizado.
Con este fin, fue necesario que la plataforma mecánica tuviera gran flexibilidad para que superara los retos que requieren realizar maniobras. Adicionalmente, el peso también fue un factor importante en el desarrollo del sistema mecánico porque, en este caso, no era necesario que el robot cuente con una amplia robustez; por el contrario, entre más liviano fuese el sistema, mejor sería el desempeño en velocidad en pista. Otra variable importante era que la superficie de la pista era alfombrada, por lo tanto, el sistema de tracción del vehículo debía ser de bastante adherencia al suelo.

De acuerdo con lo descrito anteriormente, la tabla 1 describe las variables de prioridad para puntuar lo establecido.

Tabla 1. Prioridades de las variables.

Variable

Ponderación

En esta variable se tenía en cuenta la plataforma robótica que menor

Peso peso tuviera. Se calificaba con 0 a la plataforma más pesada y con 10 a la más ligera.
Puntuación

$0-10$

$0-10$

Ejes de movimiento

En esta variable se tenía en cuenta la plataforma robótica que mejor permitiera el movimiento de los ejes. Se calificaba con 0 a la plataforma más estática y con 10 a la más versátil.

En esta variable se tenía en cuenta la plataforma robótica que

Tracción permitiera la mejor tracción (en alfombra). Se calificaba con 0 a la plataforma de menos tracción y con 10 a la de mejor tracción 
Como se puede observar en la tabla 1, se definieron tres prioridades para tener en cuenta en el sistema mecánico. Como se explicó anteriormente, la variable del peso fue la de mayor prioridad (es importante aclarar que las variables peso, ejes de movimiento y tracción eran dependientes de una variable mayor, el tiempo).

A partir de las prioridades de las variables, se tuvieron en cuenta dos modelos prácticos para el diseño del chasis mecánico. A continuación, se presentan las características principales de cada modelo mecánico, en función de las variables anteriormente descritas.

\section{Plataforma robótica con ruedas}

Las plataformas robóticas con ruedas cuentan con un chasis de estructura base donde son posicionados los ejes que se distribuyen hacia las ruedas. Por su parte, el peso de una plataforma varía según el material con el cual se construyó (normalmente, se utilizan plataformas en aluminio, debido a que este es un material maleable y ligero). La tracción que ofrece un vehículo de ruedas depende íntegramente del diámetro de estas, del ancho, del material y del diseño de la superficie, es decir, el patrón que tiene grabado la rueda.

En el caso del eje de movimiento para el vehículo con ruedas, se pueden realizar movimientos sobre su mismo eje, giros y vueltas, de ser necesario para la competencia.

Figura 5. Plataforma con seis ruedas.

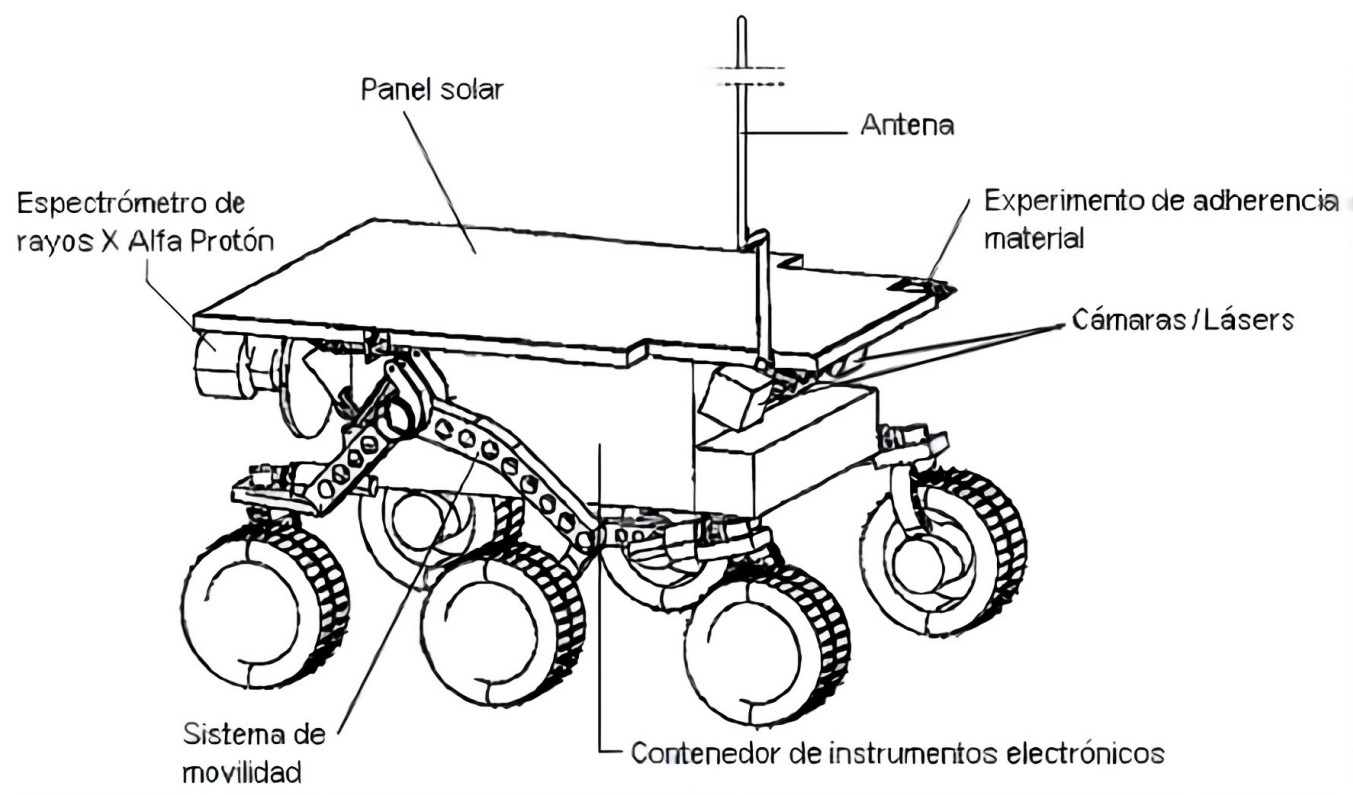

Fuente: Puentes, Ríos y Salvador (2010, p. 19).

De acuerdo con lo anteriormente descrito, en la tabla 2 se evalúa la ponderación: 
Tabla 2. Puntuación de la plataforma robótica con ruedas.

\begin{tabular}{cc} 
Variable & Puntuación \\
Peso & 5 \\
\hline Tracción & 10 \\
Total & 5 \\
\hline
\end{tabular}

Fuente: elaboración propia.

A partir de la tabla 2, es posible afirmar que en el modelo de la plataforma robótica con ruedas la tracción es relativamente baja, en relación con la que se busca para el sistema mecánico.

\section{Plataforma robótica tipo oruga}

La transmisión tipo oruga es un tipo de repartición acoplada entre el eje del motor y un piñón principal. Normalmente, se encuentra esta repartición se conecta por medio de una banda o correa a un juego de piñones encajados en el sistema de transmisión. Este tipo de transmisión de avance es óptimo para terrenos blandos, debido a que posee una tracción bastante alta, además de mejorar la distribución del peso del vehículo. De igual modo, se puede configurar para que realice movimientos sobre su mismo eje, desplazamientos hacia adelante y hacia atrás, y, en caso de necesitar mantenimiento, su reparación no es tediosa.

El peso que puede tener una plataforma tipo oruga depende de los materiales con los que se construya; en este caso, al igual que en el modelo de la plataforma con rueda, se considera una estructura en aluminio liviano, por lo tanto, la variable del peso tendrá la misma calificación que la plataforma anterior.

Figura 6. Plataforma tipo oruga.

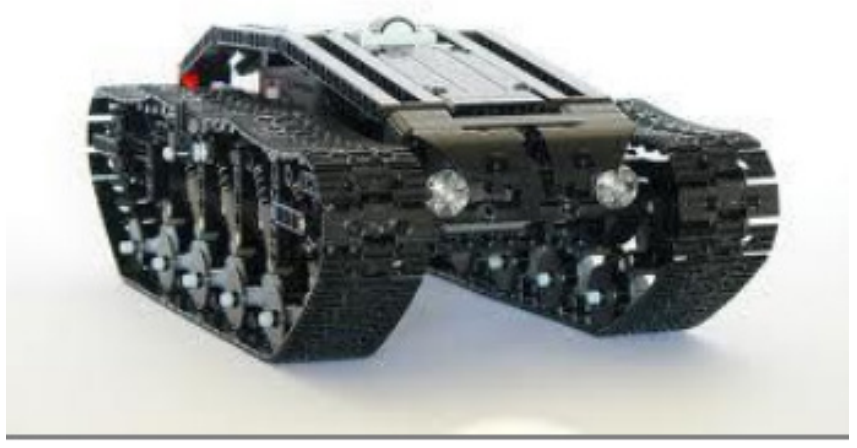

Fuente: Arboleda y Toro (2013, p. 18). 
De acuerdo con lo anteriormente descrito, en la tabla 3 se evalúa la ponderación:

Tabla 3. Puntuación de la plataforma tipo oruga.

\begin{tabular}{cc} 
Variable & Puntuación \\
Peso & 5 \\
\hline Eje de movimiento & 10 \\
Tracción & 8 \\
\hline Total & 23 \\
\hline
\end{tabular}

Fuente: elaboración propia.

Con lo observado en la tabla 3 , se puede afirmar que la tracción en la plataforma tipo oruga es ideal para el desarrollo de la competencia. Además, al tener en cuenta que la pista estaba compuesta por una alfombra, es claro que este sistema de transmisión era ideal para este proyecto.

Ahora bien, a pesar de lo observado a través de la evaluación de los diseños de las plataformas robóticas, en la elaboración del robot se decidió construir y utilizara las dos plataformas robóticas para, de un lado, poder compararlas en un ejercicio práctico y, del otro, tener mayores posibilidades de ganar el Mercury Remote Robot Challenge.

\section{Segunda parte (diseño electrónico)}

Debido a los retos de la pista, era importante tener en cuenta que la plataforma robótica debía contar con un sistema electrónico robusto, empezando por los motores que utilizaría el robot para su desplazamiento. Con este fin, en el mercado actual se encontraron motorreductores a 12 voltios DC con 360 revoluciones por minuto que contaban con un torque apropiado para la carga del robot y la superación de los obstáculos.

Mediante el uso de un puente $\mathrm{H}$-es decir, un inversor de giro para los motores- era posible que el robot se desplazara hacia cualquier dirección.

Figura 7. Puente $\mathrm{H}$ de los motores.

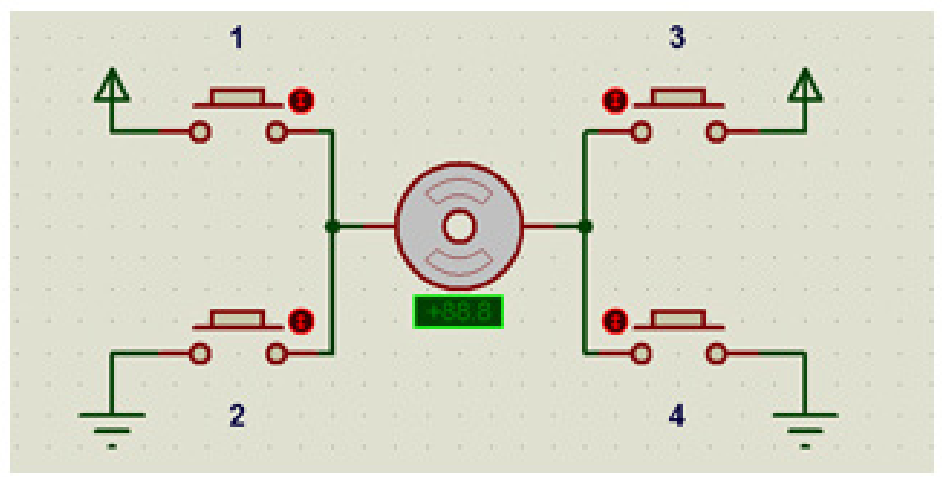

Fuente: elaboración propia. 
Toda vez que el robot debía superar los obstáculos de manera autónoma, los sensores por utilizar eran de ultrasonido; para utilizarlos, fue necesario contar con un controlador que ejecutaba las ordenes predispuestas en el software previamente cargado.

El sensor de ultrasonido de alta calidad SRF04 es capaz de calcular objetos y la distancia a la que se encuentra, en un rango de 3 a 300 centímetros, además se destaca por su bajo consumo y gran precisión.
Entre las características del sensor ultrasonido en condiciones de trabajo, se destaca el hecho de tener una gran precisión en la detección de objetos a distancia. En la figura 8 se observa el rango en grados $v s$ distancia en centímetros con el que el sensor SRF04 de ultrasonido puede usarse para el robot, con el fin de utilizar el modo autónomo en algunos obstáculos solicitados en el Mercury Remote Robot Challenge.

Figura 8. Rango de detección del sensor ultrasonido.

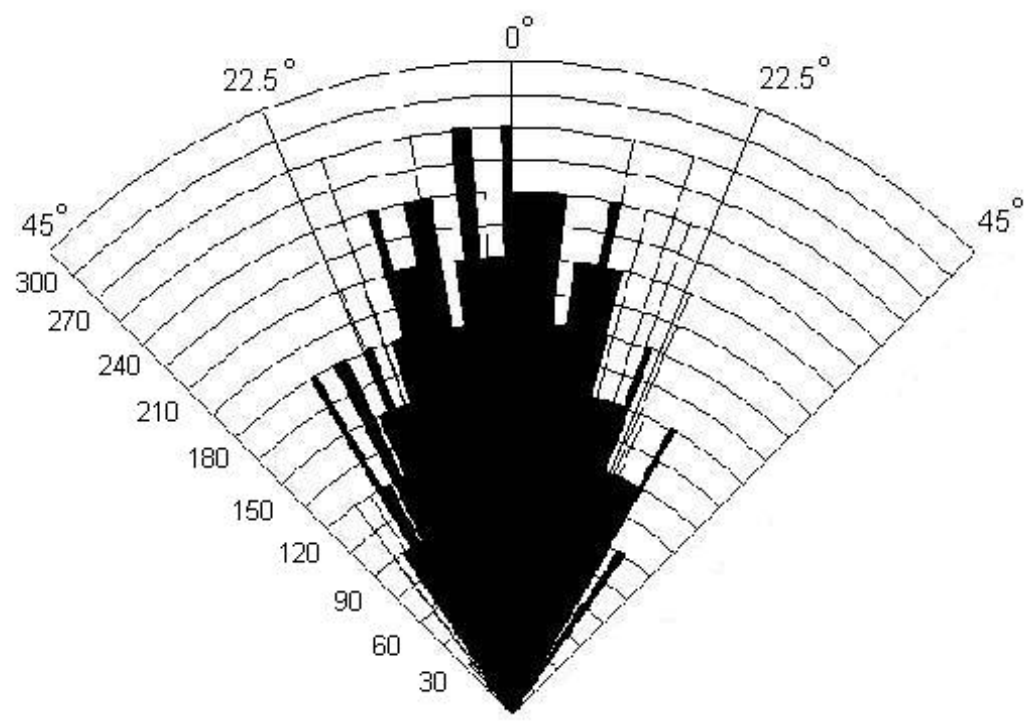

Fuente: Intplus (s. f.).

Para el sistema de comunicación RC, la organización del Mercury Remote Robot Challenge. dispuso para los participantes un canal dedicado en protocolo de Internet bajo la configuración de un canal en el router. Para establecer comunicación inalámbrica con el router en sitio, se utilizó un módulo ESP8266, que permite la conexión entre el robot y el piloto vía wifi.Por otra parte, como se evidencia en la figura 9, el diagrama de bloques permite observar el diseño electrónico para la construcción de los robots teleoperados. En la fase 1 , fue correspondiente al sistema mecánico, donde se utilizaron los dos modelos presentados (transmisión ruedas y transmisión oruga) y, además, se presenta el diseño y las piezas necesarias para el sistema electrónico. 
Figura 9. Diagrama de bloques del sistema electrónico.

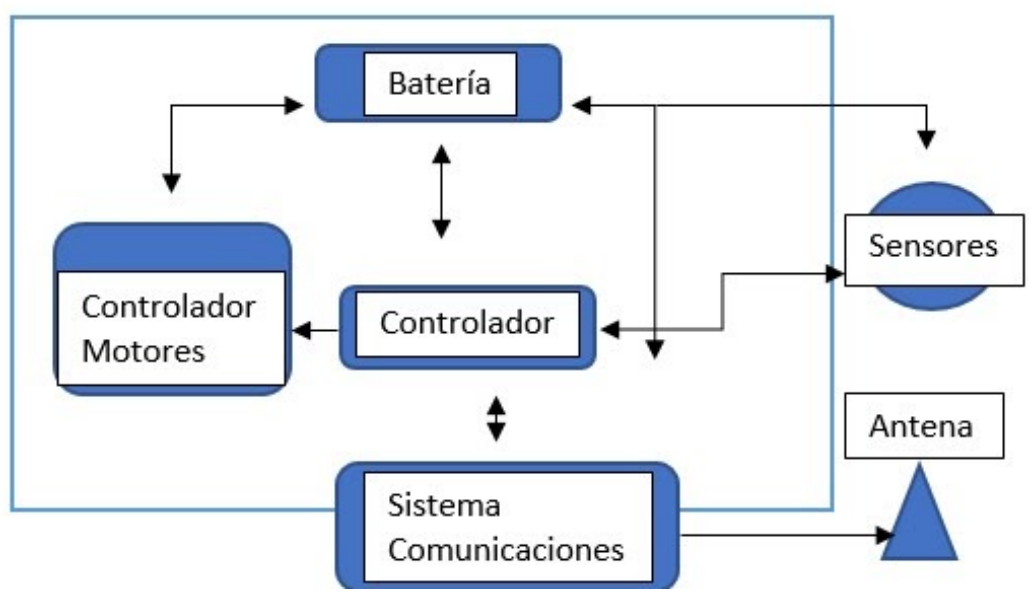

Fuente: elaboración propia.

\section{Fase 2. Construcción del prototipo}

En este apartado se presta especial atención a todo el proceso llevado a cabo para la construcción de las plataformas robóticas, evidenciando cómo fue el proceso para realizar los robots de competencia. En primer lugar, se presenta el desarrollo del sistema mecánico y las piezas que fueron fundamentales para completar la estructura o chasis que dio forma a los robots. De igual modo, se integra el sistema electrónico, para el cual se evidencian las placas realizadas para el control de los robots.

Como se mencionó anteriormente, se tomó la decisión de desarrollar dos sistemas mecánicos: uno con transmisión de ruedas y otro con transmisión tipo oruga. A continuación, se muestra cómo se desarrolló el chasis de transmisión de ruedas.

El desarrollo de esta plataforma se llevó a cabo en material de aluminio liviano, con el fin de reducir al máximo el peso de la estructura. Para esto, se realizaron diseños sobre software especializado, que permite la manipulación y construcción de piezas a escala, dando como resultado una estructura sólida y de excelente resistencia para los obstáculos que se podían presentar en la competencia.

En la figura 10 se puede apreciar cómo el robot empieza a tomar forma con la unión de las piezas del soporte de los motores y una cama baja de aluminio, donde estaría toda la electrónica del robot.

Figura 10. Plataforma robótica con motores.

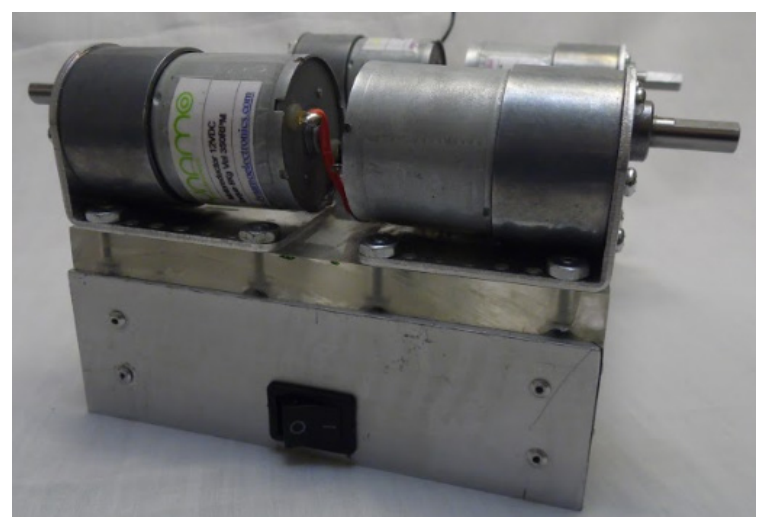

Fuente: elaboración propia.

En la figura 11 se puede evidenciar el robot "Yipao", con la plataforma mecánica de ruedas. Una vez ensamblado el chasis, se procedió a realizar el acoplamiento del sistema electrónico. 
Figura 11. Plataforma robótica con ruedas.

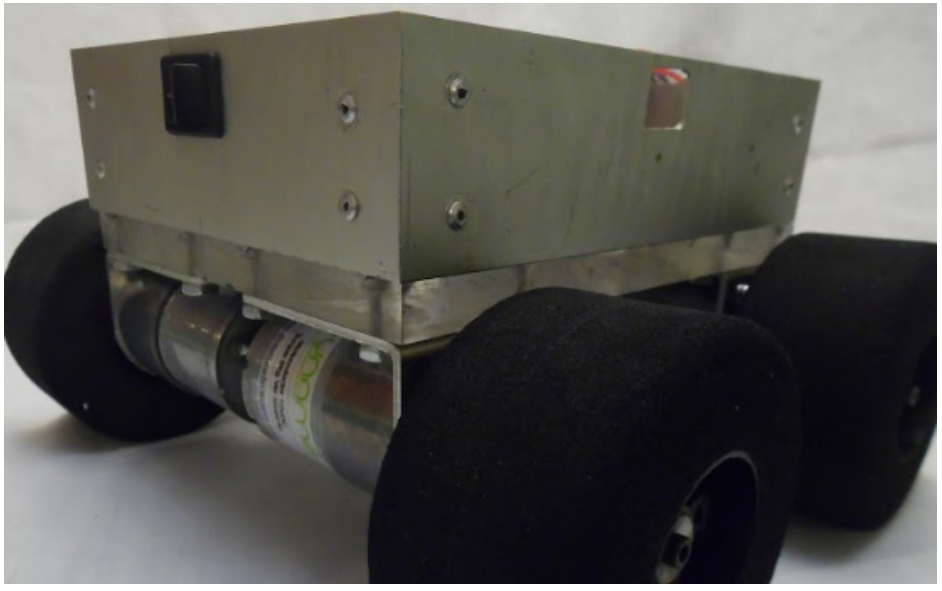

Fuente: elaboración propia.

Para la segunda plataforma robótica, con sistema de transmisión de oruga, se evidencia el desarrollo su desarrollo en la figura 12. Como se puede ver, la plataforma se realizó con una placa de acrílico y aluminio. Los motores utilizados en este modelo son iguales a los descritos anteriormente: "Socco" utiliza el mismo sistema electrónico que "Yipao". Por lo tanto, se asumió que la carga que debían tener los motores era igual a la del diseño anterior.

Figura 12. Plataforma robótica tipo oruga.

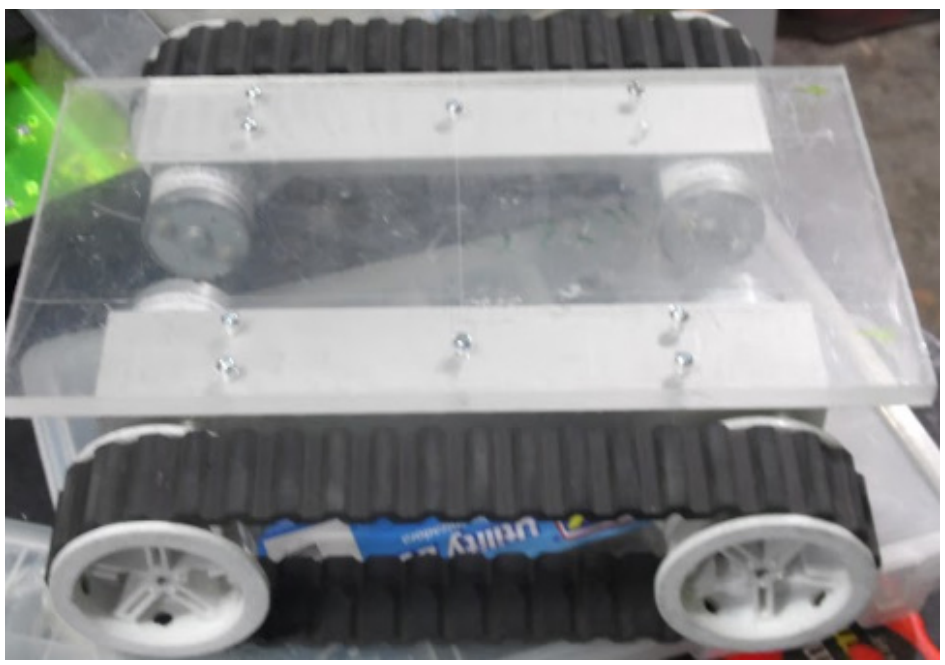

Fuente: elaboración propia.

Una vez desarrolladas las plataformas robóticas, se procedió a construir el sistema electrónico. Para este proceso, se utilizó una placa de dos caras que integra el puente $\mathrm{H}$ (control motores), el sistema de comunicación, el controlador y la conexión para los sensores. 
El desarrollo de las tarjetas electrónicas se realizó en secciones diferentes: en la primera, se desarrolló el controlador que se encargaría de enviar las señales de control al driver de los motores, adquisición y envío de datos a los sensores, y manejo de protocolo de datos de comunicación; en la segunda, se desarrolló el driver de los motores y módulo de comunicación.
Como se puede observar en la figura 13, se construyeron las placas electrónicas para la participación de los robots en el evento. El puente H para el giro de los motores se diseñó a una corriente pico de 3.5 amperios y una corriente nominal de 2 amperios. Es importante destacar que todo fue construido por el grupo del semillero del programa de ingeniería electrónica ELECTROCUN.

Figura 13. Tarjeta de control de los robots.

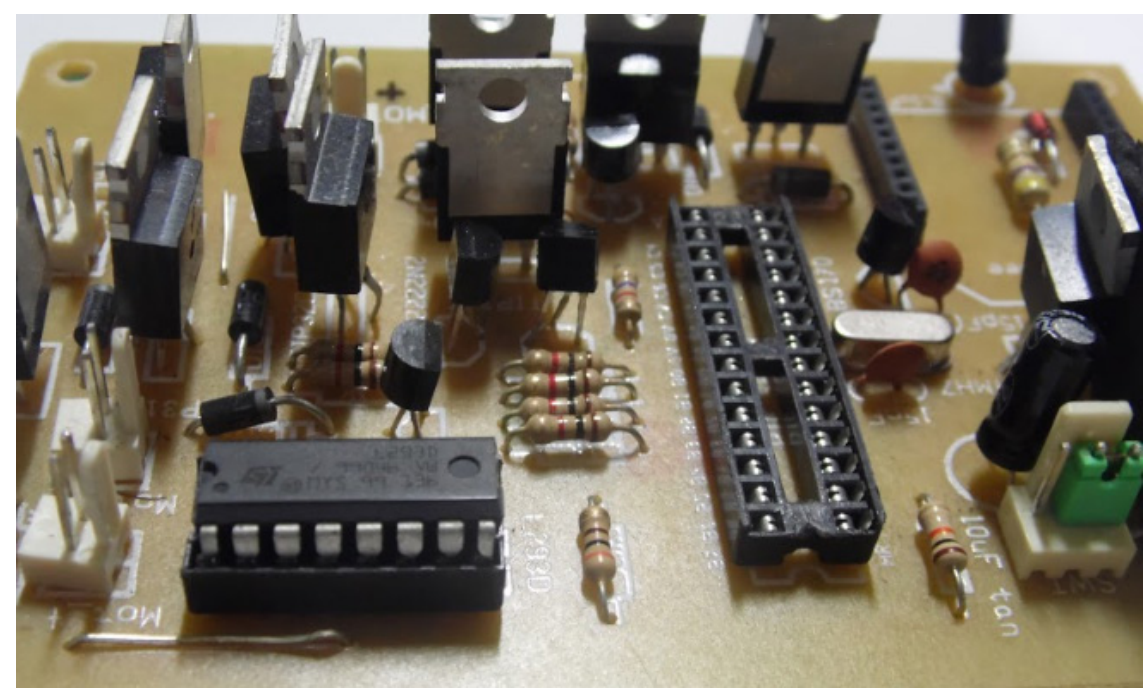

Fuente: elaboración propia.

Una vez soldados los componentes electrónicos a las tarjetas, se procedió a armar completamente los robots para realizar las pruebas correspondientes. Se utilizó una batería de

12 voltios a 3600 miliAmperios con una descarga de 25 c.

La cámara en el robot es utilizada por el piloto para observar los obstáculos y el desplazamiento del robot al recorrer la pista. La imagen de la cámara es transmitida bajo el mismo protocolo de comunicación para teleoperar al robot.
Figura 14. Robot "Yipa”" completo.

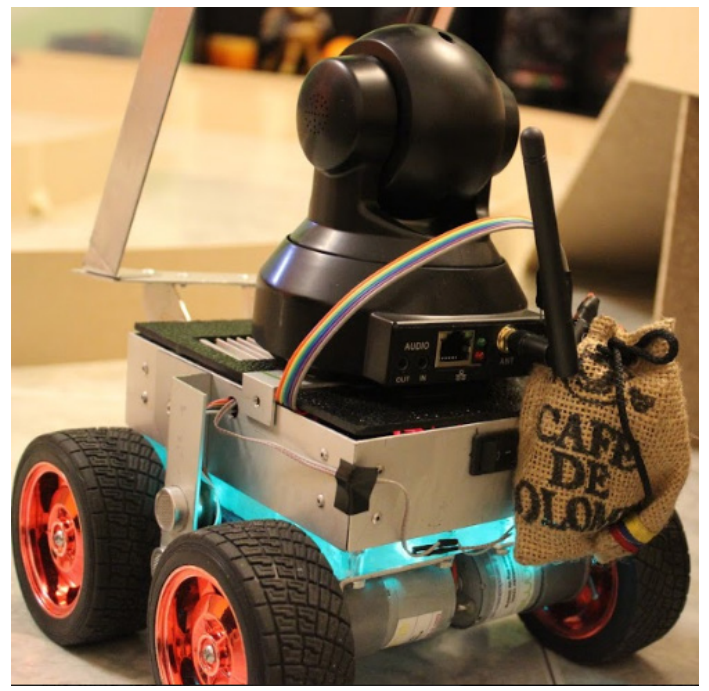

Fuente: elaboración propia. 
Finalmente, en la figura 15 se puede observar al robot "Socco" completamente ensamblado. Para la construcción de los dos robots de competencia se utilizaron materiales exactamente iguales.

Figura 15. Robot "Socco" completo.

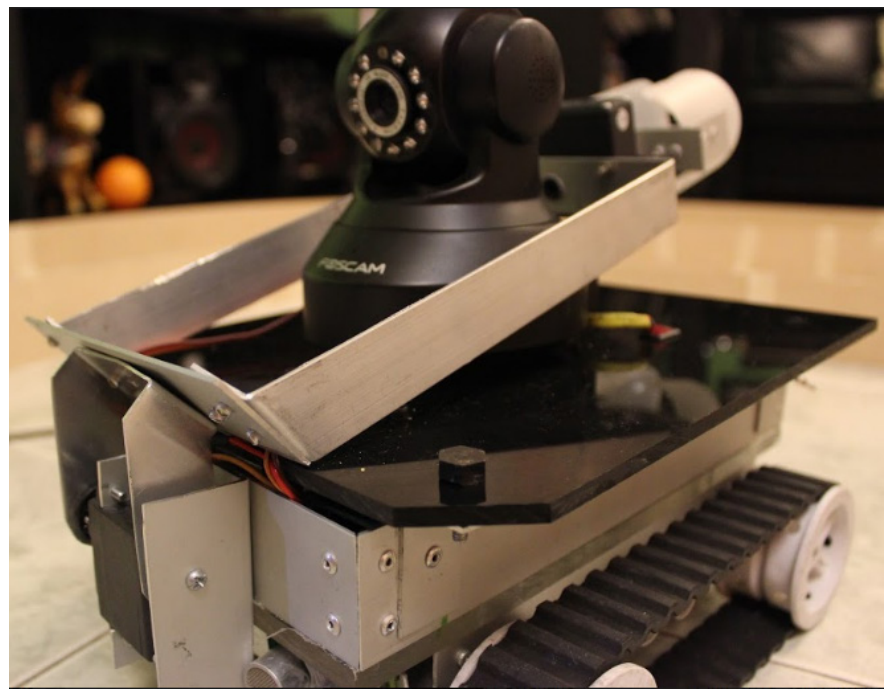

Fuente: elaboración propia.

\section{Fase 3. Evaluación de los robots en competencia}

La participación de los robots teleoperados se realizó en la Universidad Estatal de Oklahoma, Estados Unidos, donde se estableció que los pilotos operaran al robot desde Bogotá, Colombia. De esta manera, se cumplió la regla principal del evento: manejar al robot desde una distancia mínima de 80 kilómetros.

En la participación en pista, los robots "Socco" y "Yipao" se destacaron por su gran actuación y cumplimiento de los obstáculos. También se debe valorar la velocidad que tardaron en cruzar el circuito, gracias a la destreza del piloto. Con la plataforma teleoperada "Yipao" se obtuvo el primer lugar, lo que permitió el reconocimiento al grupo del semillero de investigación ELECTROCuN. Sobre un total de 150 puntos, se obtuvieron 145, y el tiempo del robot en cruzar toda la pista fue de 1 minuto, 45 segundos y 37 centésimas (1:45:37).
Figura 16. Placa obtenida por el primer puesto del robot "Yipao" en el Mercury Remote Robot Challenge.

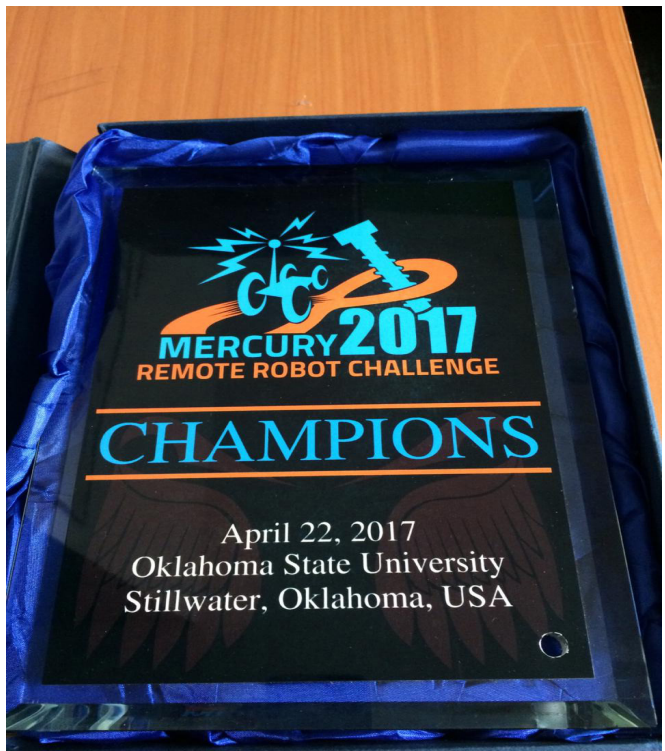

Fuente: elaboración propia.

Por su parte, el robot "Socco" también tuvo un gran desempeño en pista, pero, desafortunadamente, fue necesario realizar un arreglo sobre el 
robot durante competencia, debido a que, por cuestiones mecánicas, se desajusto el ángulo de la cámara; esto ocasionaba que el piloto no pudiese tener plena visión de la pista ni de los obstáculos. Este inconveniente se presentó faltando solamente dos obstáculos para terminar el recorrido, lo que permitió que obtuviera 138 puntos sobre los 150 posibles. El tiempo final del recorrido fue 1 minuto, 47 segundo y 42 centésimas. Este tiempo permitió que el robot lograra el segundo lugar en la competencia.

Figura 17. Placa obtenida por el segundo puesto del robot "Socco" en el Mercury Remote Robot Challenge.

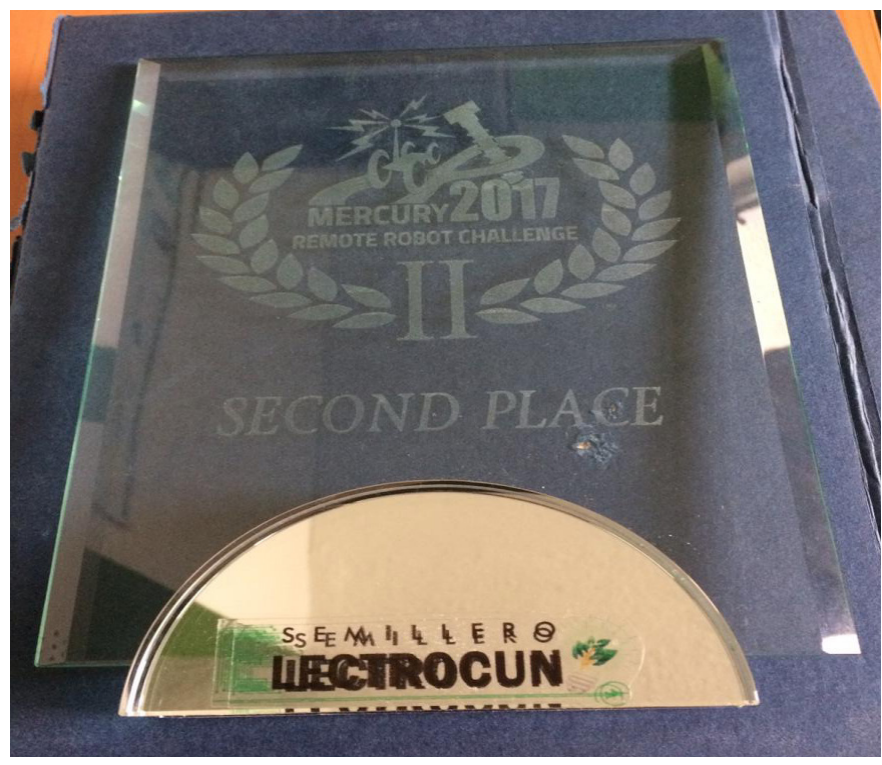

Fuente: elaboración propia.

\section{Conclusiones}

Para el semillero de investigación ELECTROCUN se destaca el hecho del trabajo en equipo; sin la unión de múltiples complementos entre profesores y estudiantes no habría sido lograr el primer y el segundo puesto del Mercury Remote Robotic Challenge. También es importante destacar el valor de apoyo a la Corporación Unificada Nacional de Educación Superior (CUN) por hacer posible la realización de los robots con la financiación de los recursos económicos.

En cuanto a las plataformas robóticas realizadas mediante múltiples ensayos y correcciones a los prototipos que se desarrollaron, se estableció que poseer un mejor sistema de comunicación permite tener una ventaja significativa, debido a que puede transmitir la información de forma rápida y eficaz al punto del router.

En las pruebas realizadas en pista, se observó que los comportamientos de los robots en sus sistemas mecánicos no tuvieron mayores complicaciones. Según lo observado en la competencia, es más rentable desarrollar el chasis en aluminio liviano que en acrílico, pues este último, luego de cierto tiempo, tiende a pandearse con el peso del robot y puede llegar a afectar el libre avance de las ruedas. 
Gracias a la experiencia en el Mercury Remote Robotic Challenge, fue posible identificar otro aspecto importante que se debe de tener en cuenta para realizar un robot teleoperado: los sensores deben ser de gran precisión y se deben calibrar en la pista, previamente al momento de la competencia, ya que algunas secciones de la pista pueden ser desiguales y no coincidir con lo mencionado en los documentos o datos previos. A modo de ejemplo, en el caso del Mercury Remote Robotic Challenge era preferible medir la distancia de algunos objetos con un sensor ultrasónico y no con un sensor óptico, porque existía un desajuste provocado por la luz artificial de la competencia, el cual no permitía que el sensor del robot trabajara óptimamente.

Finalmente, de esta experiencia fue posible aprender, de un lado, que es ideal tener bastante tiempo antes de una competencia, toda vez que, en caso de necesitarse un ajuste inesperado, sería posible trabajar junto a la pista; de otro lado, es preciso que el piloto se encuentre en un lugar sin distracciones y que cuente con un canal de acceso a Internet dedicado.

\section{Referencias}

Arboleda, A. y Toro, D. (2013). Diseño de robot tipo oruga con brazo articulado (trabajo de grado). Universidad Tecnológica de Pereira, Pereira. Recuperado de https:/ / bit.ly/2VbQUGS

Global Security. (s. f.). Gladiator Tactical Unmanned Ground Vehicle. Recuperado de https:// bit.ly/3rCX9zx

Milrem Robotics. (s. f.). Multiscope 3D. Recuperado de https:/ / bit.ly/3y6Pcot

Prometec. (s. f.). Tipos de drones. Recuperado de https:/ / bit.ly/3x0XRYo

Puentes, D., Ríos, S. y Salvador, D. (2010). Diseño e implementación de un mando de control para un vehículo teleoperado (trabajo de grado). Universidad de San Buenaventura, Bogotá. Recuperado de https://bit.ly/3kZzpEg

Super Robótica. (s. f.). SRF04 sensor distancias por ultrasonidos SRF04 s320110. Recuperado de https://bit.ly/2ULAV2q 